Provided for non-commercial research and education use. Not for reproduction, distribution or commercial use.

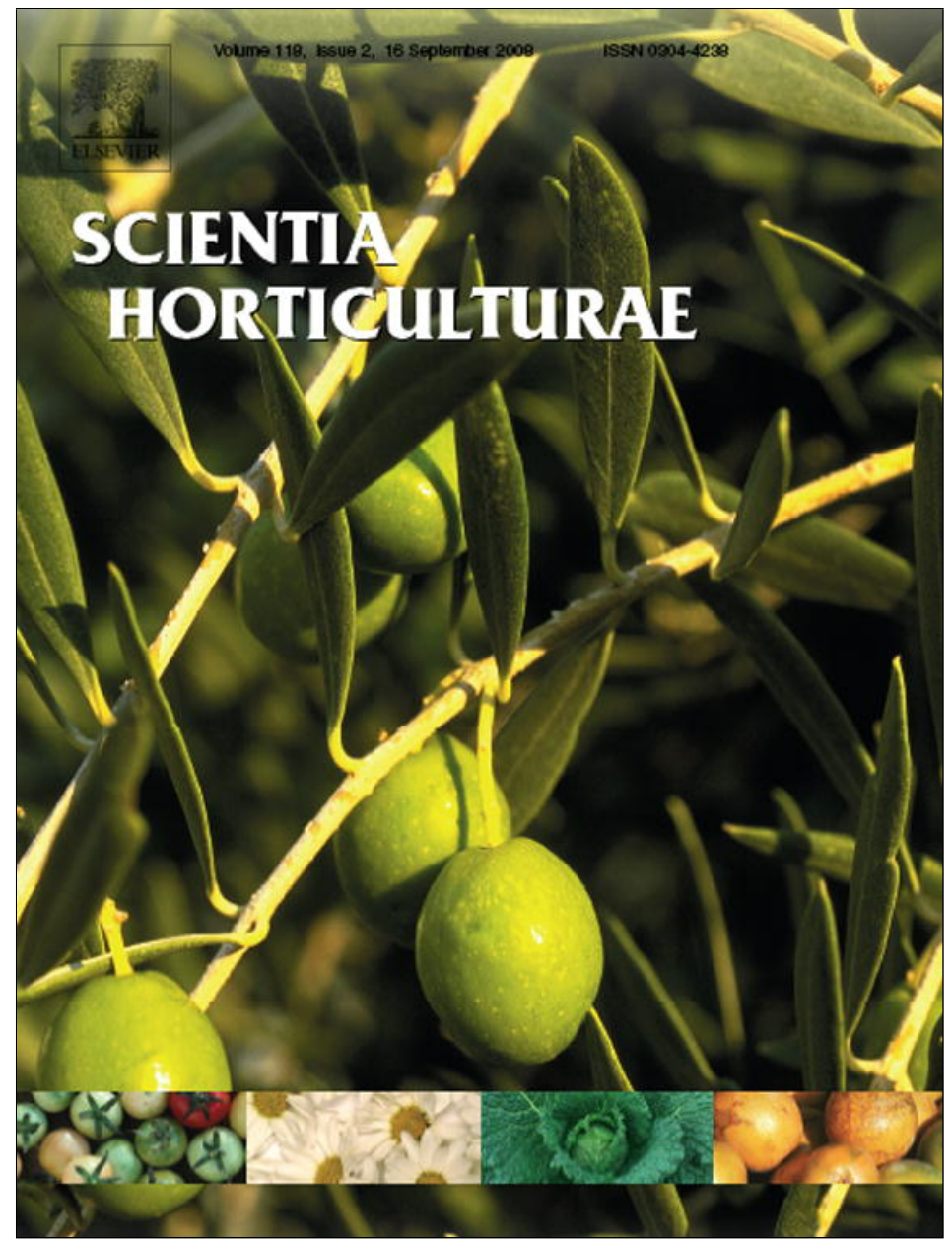

This article appeared in a journal published by Elsevier. The attached copy is furnished to the author for internal non-commercial research and education use, including for instruction at the authors institution and sharing with colleagues.

Other uses, including reproduction and distribution, or selling or licensing copies, or posting to personal, institutional or third party websites are prohibited.

In most cases authors are permitted to post their version of the article (e.g. in Word or Tex form) to their personal website or institutional repository. Authors requiring further information regarding Elsevier's archiving and manuscript policies are encouraged to visit:

http://www.elsevier.com/copyright 


\title{
Cultivar influence on virgin olive (Olea europea L.) oil flavor based on aromatic compounds and sensorial profile
}

\author{
Debora Tura $^{\mathrm{a}, *}$, Osvaldo Failla ${ }^{\mathrm{a}}$, Daniele Bassi ${ }^{\mathrm{a}}$, Stefano Pedò ${ }^{\mathrm{b}}$, Arnaldo Serraiocco $^{\mathrm{c}}$ \\ a University of Milan, Dipartimento di Produzione Vegetale (DIPROVE), Via Celoria 2, 20133 Milano, Italy \\ ${ }^{\mathrm{b}}$ Fondazione Edmund Mach, Istituto Agrario San Michele all'Adige, Via Mach 1, 38010 S. Michele all'Adige (TN), Italy \\ ${ }^{\mathrm{c}}$ CRA, Centro Centro di ricerca per l'olivicoltura e l'industria olearia, Viale Petruzzi 75, 65013 Città S. Angelo (PE), Italy
}

\section{A R T I C L E I N F O}

\section{Article history:}

Received 29 February 2008

Received in revised form 16 May 2008

Accepted 28 May 2008

\section{Keywords:}

Quality

Phenols

Volatiles

Aroma

Panel test

Head-space

\begin{abstract}
A B S T R A C T
Sensory quality is an important property of virgin olive oil and is affected by different volatile and phenolic compounds. Their levels may be influenced by many factors, and one of the most important is the cultivar. Volatiles and phenols were correlated to sensory notes in virgin olive oils from 18 local cultivars in northern Italy assessed for 4 years in the same orchard.

Most of the volatile and phenolic compounds showed an average content higher than odor and taste thresholds, explaining the correlations to sensory attributes. Some volatile compounds (e.g. ethanol, 2methyl-propan-1-ol, pentan-1-ol, cis-2-penten-1-ol, cis-3-hexen-1-ol and octan-1-ol) and sensory attributes (e.g. 'flowers', 'banana', 'apple', 'walnut', 'hay', 'butter', 'sweet', 'floral' and 'fruity' notes) were found as cultivar dependent. Some cultivars, with a similar aromatic content, showed also analogous sensorial profile. 'Favarol', 'Casaliva 1', 'Raza', 'Casaliva 2', 'Gargnà', 'Mitria', 'Miniol', and 'Rossanello' resulted in the same aromatic group, characterized by an average volatiles content, and in three close affinity groups with a middle sensorial profile. Moreover, 'Maurino' was always isolated, showing peculiar profiles. Local cultivars 'Casaliva 1', 'Cornarol', 'Grignano', 'Trepp' and 'Regina' overall have shown peculiar flavor profiles. Therefore, some of these often-underutilized cultivars could be employed in the new orchards in order to take advantage of their superior oil quality traits, in addition to some positive horticultural aspects. This could be particularly crucial for the Protected Designation of Origin (PDO) oils, where the mere geographical origin could not be enough in favoring olive oil characterization and consumption, if sensorial and/or nutritional attributes are also not differentiated within a standard commercial commodity.
\end{abstract}

(c) 2008 Elsevier B.V. All rights reserved.

\section{Introduction}

The extra virgin olive oil is the principal source of fat in the Mediterranean diet with important nutraceutic effects due to its abundance of oleic acid, a monounsaturated fatty acid controlling the cholesterol level, and an adequate content of linoleic and linolenic acids, the major essential fatty acids that lower the risk of coronary heart diseases and cancers (Galli and Visioli, 1999). Virgin olive oil is the only vegetal fat that can be eaten crude (also called "olive juice") with no refining operations. This allows to preserve its natural composition, including the minor, non-saponificable compounds, making up to 1-2\% of total content, e.g. hydrocarbons, phenols, alcohols, sterols, pigments, tocopherols and vitamins.

\footnotetext{
* Corresponding author. Tel.: +39 02503 16557; fax: +39 0250316553. E-mail address: Debora.Tura@unimi.it (D. Tura).
}

These compounds are crucial both for the oil oxidative stability (improving the shelf life) and for its unique flavor. Aroma and taste are the only parameters that consumers can appraise directly, while other quality features (e.g. chemical composition) are not always labelled on the bottle.

Two recent works reviewed the several factors influencing aromatic quality of virgin olive oil, i.e. biogenesis and composition of volatiles, relationships with sensory notes, possible influence of agronomic and processing factors, and oil oxidation (Kalua et al., 2007; Angerosa et al., 2004). All these findings show that volatiles content, mainly $\mathrm{C}_{6^{-}}$and $\mathrm{C}_{5}$-skeleton compounds from the lipoxygenate pathway, are strongly influenced by the genetic origin (cultivar) for the enzymatic expression and by horticultural and processing parameters for the enzyme activity. The unique flavor of virgin olive oil is mainly attributed to the volatiles that develop during and after oil extraction from the fruit. These compounds become less important during oil storage due to 
oxidation. These changes on volatile composition, together with genetic, horticultural and processing influences, explain quality differences in olive oils (Harwood and Aparicio, 2000).

In a previous study (Tura et al., 2007), the antioxidant profile was found to be firstly influenced by the cultivar, and then by the site of cultivation. The oxidative stability was correlated to phenols, tocopherols and saturated on unsaturated fatty acids.

In another study several olive accessions from a local cultivars collection in the lake Garda area (northern Italy) were successfully classified by oil sensory profile (Tura et al., 2002). Moreover, these sensorial attributes resulted correlated to some volatile and phenolic compounds. A similar cultivar assessment was scored by a principal components analysis and eight aromatic compounds were found to be the more significant in varietal characterization, e.g. cis-3-hexen-1-ol, hexan-1-ol, pentan-3-one and trans-2hexen-1-ol (Pedò et al., 2002). Furthermore, an in-depth study on the two main cultivars in the above region found that on the basis of a similar fruit ripening stage, the 'Leccino' oil showed a higher aromatic compounds content compared to 'Casaliva'. But due to local practice, 'Leccino' is usually harvested at full maturation ('black' stage), when most volatiles are gone and antioxidants are low, while 'Casaliva' is picked at an early stage, even before the veraison, in order to avoid oil being negatively affected by possible early winter frost on fruits. This is why commercial 'Leccino' oil, very often, is less flavored and has lower antioxidant attribute than 'Casaliva' (Pedò et al., 2003). Thus, the olive ripening stage at harvest is a crucial step in determining the oil quality. Other findings (Tura et al., in press, 2005) confirmed that the volatile compounds in 'Casaliva' and 'Leccino' oils are highly and negatively correlated to the maturity stage of the fruits, as already shown in the above report. Mere in detail, 'Casaliva' oils were also affected by the season thermal course during ripening: oils from olives at the same maturity stage were higher in phenols, tocopherols and volatiles in years with higher heat summation.

A study on volatile profile of Australian virgin olive oils has shown cultivar as the single-most important factor in determining aromatic oil quality (Tura et al., 2004). Other works confirmed the cultivar strong effect on aromatic quality. Dhifi et al. (2005) found that the different volatile composition in four Tunisian in oils was affected by the cultivar, showing also a close relation to the enzymatic profiling, that is genetically determined. Luna et al. (2006) characterized many virgin olive oils from several countries by volatile compounds and the sensory attributes. Berlioz et al. (2006) analyzed the volatile and flavor compositions of several French oils from Protected Designation of Origin (PDO) districts and standard commercial olive oils, developing a chemometric method able to discriminate the oils. Baccouri et al. (2007) demonstrated that the volatile profiles of oleaster oils (olive cultivars selected from wild olives, Olea europea var. oleaster) were different from standard European and Tunisian virgin oils from Olea europea var. sativa. Other peculiar differences in the composition of volatile in Tunisian and French PDO oils were found by Haddada et al. (2007) demonstrating that the building up of metabolites in oils from different cultivars was related to genetic origin. Vichi et al. (2003) found significant differences in volatile composition in oils of different cultivars and geographical origin in northern Italy.

Some authors compared different analytical techniques to assess the volatile compounds and/or sensory attributes in olive oils. Procida et al. (2005) arranged a chemometric approach to correlate volatile molecules with oil sensory defects. Also Morales et al. (2005) studied the correlations between volatile profiles and defects by olfactometry techniques. Cavalli et al. (2003) and Kanavouras et al. (2005) compared several methods for volatiles extraction from oils, i.e. static and dynamic headspace, solid phase micro-extraction (SPME), sorptive extraction and thermal deso- rption. Garcia-Gonzalez et al. (2004) tested the electronic nose coupled with SPME to distinguish different olive oils. Contini and Esti (2006) checked the effectiveness of HS-SPME for the volatiles analysis of virgin olive oils at different dilutions and Jimenez et al. (2006) applied this method to carry out a quality control of virgin olive oils from fruit picked either from the tree or from the ground.

In this paper, we have investigated the volatile composition and the sensory notes of monovarietals oils from local olive cultivars for an in-depth study of their aromatic pattern (Alfei, 2004). The main goal of this work was to deeply investigate the potential of oftenneglected germoplasm at risk of extinction that could play a crucial role in improving the flavor attributes of commercial products. There is an increasing interest on extraction olive oil but there is a strong risk this demand is not matched by high quality products.

\section{Materials and methods}

\subsection{Oils sampling}

The study was carried for 4 years on oil samples obtained from 18 olive cultivars grown in the same orchard in the western coast of the Garda lake (northern Italy). The orchard is located in Raffa di Puegnago at $170 \mathrm{~m}$ a.s.l., $45^{\circ} 32^{\prime} \mathrm{N}$ of latitude and $10^{\circ} 31^{\prime} \mathrm{E}$ of longitude, its landscape being a coarse soil on moraine foothill with an annual mean temperature and rainfall of $13.6{ }^{\circ} \mathrm{C}$ and $937 \mathrm{~mm}$, respectively. All oil samples were obtained from about $10 \mathrm{~kg}$ of olives at veraison stage by a standard discontinuous procedure within 1 day from picking. The olives were crushed with a stainless steel hammer crusher mill and malaxed for $30 \mathrm{~min}$ at $28^{\circ} \mathrm{C}$. The oil was extracted by hydraulic press (maximum $20 \mathrm{MPa}$ ) and

Table 1

Oil aromatic profiling (3-4 years average): number of samples, value range, mean, standard deviation and expected variance component due to cultivar and its interactions

\begin{tabular}{|c|c|c|c|c|c|}
\hline Compound & $N$ & $\begin{array}{l}\text { Range } \\
(\mathrm{mg} / \mathrm{kg})\end{array}$ & $\begin{array}{l}\text { Mean } \\
(\mathrm{mg} / \mathrm{kg})\end{array}$ & $\begin{array}{l}\text { S.D. } \\
(\mathrm{mg} / \mathrm{kg})\end{array}$ & $\begin{array}{l}\text { Variance } \\
\text { (\%) }\end{array}$ \\
\hline n-Octane & 60 & $0.33-33.3$ & 3.39 & n.r. & 25 \\
\hline Ethyl acetate & 60 & $0.28-80.9$ & 13.4 & n.r. & 0 \\
\hline 2-Methyl-butanal & 60 & $0.05-37.7$ & 0.33 & n.r. & 6 \\
\hline 3-Methyl-butanal & 60 & $0.05-43.9$ & 5.64 & n.r. & 5 \\
\hline Ethanol & 60 & $3.47-217.7$ & 30.6 & n.r. & 53 \\
\hline Pentan-3-one & 60 & $0.88-119.6$ & 20.6 & n.r. & 4 \\
\hline 1-Penten-3-one & 60 & $0.81-27.8$ & 9.54 & 5.97 & 37 \\
\hline Hexanal & 60 & $0.53-144.2$ & 35.2 & 31.8 & 27 \\
\hline 2-Methyl-propan-1-ol & 60 & $0.05-2.69$ & 0.76 & 0.59 & 76 \\
\hline trans-2-pentenal & 60 & $0.09-32.4$ & 7.48 & 6.91 & 39 \\
\hline 1-Penten-3-ol & 60 & $1.63-74.1$ & 28.7 & 19.7 & 9 \\
\hline 3-Methyl-butan-1-ol & 60 & $0.38-15.1$ & 6.14 & 3.98 & 17 \\
\hline trans-2-Hexenal & 60 & $1.25-1863.5$ & 543.4 & 514.7 & 28 \\
\hline Pentan-1-ol & 60 & $0.04-6.77$ & 1.05 & n.r. & 67 \\
\hline cis-2-Penten-1-ol & 60 & $1.61-139.9$ & 22.3 & 19.8 & 50 \\
\hline Hexan-1-ol & 60 & $1.08-89.5$ & 17.4 & n.r. & 3 \\
\hline cis-3-Hexen-1-ol & 60 & $1.83-258.6$ & 41.3 & n.r. & 93 \\
\hline trans-2-Hexen-1-ol & 60 & $0.22-304.4$ & 33.8 & n.r. & 9 \\
\hline Acetic acid & 60 & $0.04-1.14$ & 0.33 & 0.25 & 1 \\
\hline Octan-1-ol & 60 & $0.24-7.09$ & 0.70 & n.r. & 92 \\
\hline Total alcohols & 60 & $52.4-541.3$ & 182.7 & 112.8 & 6 \\
\hline Total aldehydes & 60 & $2.55-1926.4$ & 596.2 & 533.7 & 27 \\
\hline Total ketones & 60 & $3.63-127.2$ & 30.1 & 23.9 & 5 \\
\hline Total $C_{5}$ compounds & 60 & $4.54-198.8$ & 68.0 & 40.9 & 8 \\
\hline Total $\mathrm{C}_{6}$ compounds & 60 & 23.9-1998.4 & 671.1 & 543.6 & 22 \\
\hline Total $C_{6}$ from LA & 60 & $5.20-170.9$ & 52.7 & 40.2 & 12 \\
\hline Total $C_{6}$ from $\operatorname{Ln} A$ & 60 & $18.3-1942.5$ & 618.4 & 523.2 & 23 \\
\hline Total volatiles & 60 & $62.8-2184.2$ & 826.2 & 573.0 & 18 \\
\hline Total phenols ${ }^{a}$ & 61 & $55.4-615.5$ & 235.5 & 128.4 & 12 \\
\hline
\end{tabular}

n.r., not reported because frequency distributions were not normal according to Kolmogorov-Smirnov test $(P=0.05)$. LA, linoleic acid. LnA, linolenic acid.

a From Tura et al. (2007). 
Table 2

Oil aromatic profiling: comparison among 18 cultivars (within brackets: number of cropping years)

\begin{tabular}{|c|c|c|c|c|c|c|}
\hline Compound (mg/kg) & Baia (3) & Casaliva 1 (4) & Casaliva $2(4)$ & Cornarol (3) & Favarol (4) & Frantoio (4) \\
\hline$n$-Octane & $5.03 \mathrm{ab}^{\mathrm{a}}$ & $2.77 \mathrm{ab}$ & $5.52 \mathrm{ab}$ & $1.27 \mathrm{a}$ & $1.67 a b$ & $9.38 b$ \\
\hline Ethyl acetate & $7.80 \mathrm{a}$ & $6.13 a$ & $5.97 a$ & $10.09 a$ & $30.13 a$ & $16.12 a$ \\
\hline 2-Methyl-butanal & $8.55 a$ & $2.09 a$ & $2.27 \mathrm{a}$ & $6.96 a$ & $6.86 a$ & $1.58 \mathrm{a}$ \\
\hline 3-Methyl-butanal & $8.23 a$ & $2.36 a$ & $3.72 \mathrm{a}$ & $8.03 a$ & $9.49 a$ & $2.31 \mathrm{a}$ \\
\hline Ethanol & $92.35 b$ & $15.33 a$ & $22.17 a$ & $34.28 a$ & $28.75 a$ & $20.32 a$ \\
\hline Pentan-3-one & 28.98ab & 18.03ab & 21.86ab & $50.73 b$ & 28.10ab & $36.92 \mathrm{ab}$ \\
\hline 1-Penten-3-one & 13.97bcd & 7.60abc & $11.80 \mathrm{abc}$ & $12.38 \mathrm{abc}$ & 5.75ab & 8.27abc \\
\hline Hexanal & 49.51 abcd & 43.50abcd & $53.38 \mathrm{bcd}$ & 48.58abcd & $24.64 \mathrm{abc}$ & $71.96 \mathrm{~d}$ \\
\hline 2-Methyl-propan-1-ol & $1.31 \mathrm{~b}$ & $0.48 \mathrm{ab}$ & $0.59 \mathrm{ab}$ & $0.97 a b$ & $0.62 \mathrm{ab}$ & $0.59 \mathrm{ab}$ \\
\hline trans-2-Pentenal & 10.62ab & $6.81 \mathrm{a}$ & $6.62 a$ & $10.35 \mathrm{ab}$ & $4.30 \mathrm{a}$ & $7.48 a$ \\
\hline 1-Penten-3-ol & 31.01abc & 33.17abc & 36.93abc & 28.92abc & $20.41 a$ & 35.72abc \\
\hline 3-Methyl-butan-1-ol & $8.97 b$ & $8.51 b$ & 6.87ab & 4.37ab & 7.50ab & $9.16 b$ \\
\hline trans-2-Hexenal & $211.2 \mathrm{abc}$ & $1179.9 \mathrm{~g}$ & 1031.0efg & $175.8 \mathrm{ab}$ & 532.3abcdef & $1112.4 \mathrm{fg}$ \\
\hline Pentan-1-ol & $0.81 \mathrm{abc}$ & 1.45abc & $1.26 a b c$ & $0.60 \mathrm{abc}$ & $0.31 \mathrm{a}$ & $1.41 \mathrm{abc}$ \\
\hline cis-2-Penten-1-ol & $25.08 \mathrm{a}$ & $21.38 a$ & $21.97 a$ & $27.02 \mathrm{a}$ & $15.35 a$ & $20.37 a$ \\
\hline Hexan-1-ol & $16.14 a$ & $9.67 a$ & $28.86 a$ & $21.27 a$ & $34.57 a$ & $26.41 a$ \\
\hline cis-3-Hexen-1-ol & $83.88 \mathrm{bcd}$ & $15.83 \mathrm{ab}$ & $25.52 \mathrm{ab}$ & $139.13 d$ & 12.39ab & 13.12ab \\
\hline trans-2-Hexen-1-ol & $17.74 a$ & $34.28 \mathrm{a}$ & $68.46 a b$ & $23.82 \mathrm{a}$ & 60.88ab & $55.38 \mathrm{ab}$ \\
\hline Acetic acid & $0.32 \mathrm{a}$ & $0.34 \mathrm{a}$ & $0.48 a$ & $0.34 a$ & $0.21 \mathrm{a}$ & $0.38 \mathrm{a}$ \\
\hline Octan-1-ol & $0.60 \mathrm{a}$ & $0.59 a$ & $0.56 a$ & $1.16 \mathrm{a}$ & $0.78 a$ & $0.48 a$ \\
\hline Total alcohols & $277.90 a$ & 140.69a & 213.19a & $281.53 a$ & $181.55 a$ & 182.96a \\
\hline Total aldehydes & $288.12 \mathrm{ab}$ & $1234.69 d$ & $1097.04 \mathrm{~cd}$ & 249.76ab & 577.56abcd & $1195.73 d$ \\
\hline Total ketones & $42.94 a$ & $25.62 a$ & $33.66 a$ & $63.11 a$ & $33.85 a$ & 45.19a \\
\hline Total $C_{5}$ compounds & $80.68 \mathrm{ab}$ & 68.96ab & 77.32ab & 78.67ab & $45.81 \mathrm{a}$ & 71.84ab \\
\hline Total $C_{6}$ compounds & $378.48 \mathrm{ab}$ & $1283.22 b$ & $1207.26 b$ & 408.63ab & $664.75 a b$ & $1279.27 \mathrm{~b}$ \\
\hline Total $C_{6}$ from LA & $65.66 a$ & 53.17a & $82.24 a$ & $69.85 a$ & $59.22 \mathrm{a}$ & 98.36a \\
\hline Total $C_{6}$ from $\operatorname{Ln} A$ & $312.83 \mathrm{ab}$ & $1230.05 c$ & $1125.02 \mathrm{bc}$ & $338.78 \mathrm{ab}$ & $605.53 a b c$ & $1180.90 b c$ \\
\hline Total volatiles & $622.1 \mathrm{ab}$ & $1410.3 c$ & $1355.9 c$ & $606.1 \mathrm{ab}$ & 825.0abc & $1449.8 c$ \\
\hline Total phenols ${ }^{\mathrm{b}}$ & $213.9 d$ & $131.3 b c$ & $150.0 \mathrm{c}$ & 239.3de & $128.8 \mathrm{bc}$ & $143.4 \mathrm{bc}$ \\
\hline Compound (mg/kg) & Gargnà (3) & Grignano (3) & Leccino (4) & Less (3) & Maurino (3) & Miniol (4) \\
\hline n-Octane & 3.09ab & $2.17 \mathrm{ab}$ & $1.57 \mathrm{ab}$ & $1.84 \mathrm{ab}$ & 4.97ab & $2.47 \mathrm{ab}$ \\
\hline Ethyl acetate & $30.20 a$ & $9.15 a$ & $8.08 \mathrm{a}$ & $7.75 a$ & $18.89 a$ & $18.81 \mathrm{a}$ \\
\hline 2-Methyl-butanal & $1.90 \mathrm{a}$ & $2.12 \mathrm{a}$ & $9.28 \mathrm{a}$ & $3.20 \mathrm{a}$ & $1.09 a$ & $5.30 \mathrm{a}$ \\
\hline 3-Methyl-butanal & $2.76 a$ & $2.31 \mathrm{a}$ & 14.13ab & $4.28 a$ & $0.93 a$ & $7.42 a$ \\
\hline Ethanol & $29.39 a$ & $33.79 a$ & $23.29 a$ & $30.36 a$ & $25.98 a$ & $38.42 a$ \\
\hline Pentan-3-one & $22.48 \mathrm{ab}$ & $30.27 a b$ & $21.43 a b$ & $7.53 a$ & 19.03ab & $10.33 a$ \\
\hline 1-Penten-3-one & 11.98abc & $5.05 a b$ & $9.70 \mathrm{abc}$ & $5.58 \mathrm{ab}$ & $15.30 \mathrm{~cd}$ & $5.91 \mathrm{ab}$ \\
\hline Hexanal & $21.27 a b$ & 23.64ab & $32.58 \mathrm{abcd}$ & 19.43ab & $66.18 \mathrm{~cd}$ & $10.80 \mathrm{a}$ \\
\hline 2-Methyl-propan-1-ol & $0.85 \mathrm{ab}$ & $0.80 \mathrm{ab}$ & $0.94 \mathrm{ab}$ & $0.47 \mathrm{ab}$ & $2.13 c$ & $0.81 \mathrm{ab}$ \\
\hline trans-2-Pentenal & $4.44 a$ & $6.92 a$ & $5.88 \mathrm{a}$ & $4.77 a$ & $15.51 \mathrm{bc}$ & $7.53 a$ \\
\hline 1-Penten-3-ol & $26.14 a b$ & $17.51 \mathrm{a}$ & $19.07 a$ & 25.87ab & $26.21 \mathrm{ab}$ & $19.80 \mathrm{a}$ \\
\hline 3-Methyl-butan-1-ol & 5.03ab & $8.23 \mathrm{~b}$ & 7.08ab & $6.77 a b$ & 4.03ab & $5.70 \mathrm{ab}$ \\
\hline trans-2-Hexenal & 528.3abcdef & $59.9 a$ & 790.8cdefg & 451.0abcde & 211.3abc & $42.8 \mathrm{a}$ \\
\hline Pentan-1-ol & $1.10 \mathrm{abc}$ & $2.86 \mathrm{c}$ & $0.56 a b c$ & $0.91 \mathrm{abc}$ & $2.66 b c$ & $0.55 a b$ \\
\hline cis-2-Penten-1-ol & $16.42 a$ & $65.06 \mathrm{~b}$ & $12.81 \mathrm{a}$ & $13.58 \mathrm{a}$ & $20.96 a$ & $27.16 a$ \\
\hline Hexan-1-ol & $23.36 a$ & $16.82 \mathrm{a}$ & $16.10 \mathrm{a}$ & $11.29 a$ & $23.18 a$ & $5.90 a$ \\
\hline cis-3-Hexen-1-ol & $5.34 \mathrm{a}$ & $95.91 \mathrm{~cd}$ & $12.54 \mathrm{ab}$ & $10.95 a$ & $148.32 d$ & 42.70abc \\
\hline trans-2-Hexen-1-ol & $35.45 a$ & $10.62 \mathrm{a}$ & $57.82 \mathrm{ab}$ & $11.14 a$ & $11.70 a$ & $2.63 a$ \\
\hline Acetic acid & $0.12 \mathrm{a}$ & $0.29 a$ & $0.29 a$ & $0.27 a$ & $0.47 a$ & $0.33 a$ \\
\hline Octan-1-ol & $0.57 a$ & $0.59 a$ & $0.57 a$ & $0.51 \mathrm{a}$ & $0.49 a$ & $0.47 a$ \\
\hline Total alcohols & $143.65 a$ & $252.20 a$ & $150.79 a$ & $111.85 a$ & $265.68 a$ & $144.14 a$ \\
\hline Total aldehydes & 558.72abcd & $94.85 \mathrm{ab}$ & $852.66 \mathrm{bcd}$ & 482.72abcd & $295.02 \mathrm{ab}$ & $73.83 a$ \\
\hline Total ketones & $34.46 a$ & $35.32 \mathrm{a}$ & $31.12 \mathrm{a}$ & $13.12 \mathrm{a}$ & $34.33 a$ & $16.24 a$ \\
\hline Total $C_{5}$ compounds & 58.99ab & $94.54 \mathrm{ab}$ & 47.46ab & $49.80 \mathrm{ab}$ & 77.99ab & $60.40 \mathrm{ab}$ \\
\hline Total $C_{6}$ compounds & 613.77ab & $206.84 a$ & $909.83 \mathrm{ab}$ & $503.85 a b$ & 460.69ab & $104.81 \mathrm{a}$ \\
\hline Total $C_{6}$ from LA & $44.63 a$ & $40.45 a$ & $48.68 a$ & $30.72 a$ & $89.36 a$ & $16.70 \mathrm{a}$ \\
\hline Total $C_{6}$ from $\operatorname{Ln} A$ & 569.14abc & $166.39 a$ & $861.15 a b c$ & 473.12abc & $371.32 \mathrm{abc}$ & $88.11 \mathrm{a}$ \\
\hline Total volatiles & $770.2 \mathrm{abc}$ & 394.0ab & $1044.5 b c$ & $617.5 \mathrm{ab}$ & 619.4ab & $255.8 \mathrm{a}$ \\
\hline Total phenols ${ }^{\mathrm{b}}$ & $104.1 \mathrm{ab}$ & $159.3 c$ & 250.7de & $160.4 c$ & 243.9de & $126.3 \mathrm{abc}$ \\
\hline Compound (mg/kg) & Mitria (4) & Pendolino (3) & Raza (3) & Regina (3) & Rossanello (3) & Trepp (2) \\
\hline$n$-Octane & 3.18ab & $5.02 \mathrm{ab}$ & 3.03ab & $1.09 a$ & $2.97 a b$ & $2.80 \mathrm{ab}$ \\
\hline Ethyl acetate & $25.41 \mathrm{a}$ & $7.81 \mathrm{a}$ & $6.07 a$ & $6.19 a$ & $9.31 \mathrm{a}$ & $11.46 a$ \\
\hline 2-Methyl-butanal & $1.19 a$ & $5.36 a$ & $2.21 \mathrm{a}$ & $4.80 \mathrm{a}$ & $2.65 a$ & $19.78 b$ \\
\hline 3-Methyl-butanal & $1.67 \mathrm{a}$ & $5.80 \mathrm{a}$ & $2.50 a$ & $5.37 a$ & $2.52 \mathrm{a}$ & $22.85 b$ \\
\hline Ethanol & $27.48 \mathrm{a}$ & $20.19 a$ & $26.07 a$ & $25.13 a$ & $38.18 a$ & $33.30 \mathrm{a}$ \\
\hline Pentan-3-one & $12.12 \mathrm{ab}$ & $25.12 \mathrm{ab}$ & $7.43 a$ & $11.34 a$ & $6.93 a$ & $5.91 \mathrm{a}$ \\
\hline 1-Penten-3-one & 8.08abc & 10.29abc & $9.24 \mathrm{abc}$ & $21.71 d$ & $6.35 \mathrm{abc}$ & $4.08 \mathrm{a}$ \\
\hline
\end{tabular}


Table 2 (Continued)

\begin{tabular}{|c|c|c|c|c|c|c|}
\hline Compound (mg/kg) & Mitria (4) & Pendolino (3) & Raza (3) & Regina (3) & Rossanello (3) & Trepp (2) \\
\hline Hexanal & 18.67ab & 42.29abcd & 25.03abc & 39.73abcd & 14.63ab & $20.01 \mathrm{ab}$ \\
\hline 2-Methyl-propan-1-ol & $0.37 a b$ & $0.93 a b$ & $0.45 a b$ & $0.86 a b$ & $0.38 a b$ & $0.31 a$ \\
\hline trans-2-Pentenal & $5.45 a$ & $5.24 \mathrm{a}$ & $5.94 a$ & $18.63 c$ & $4.38 \mathrm{a}$ & $6.07 a$ \\
\hline 1-Penten-3-ol & $45.05 b c$ & $13.48 \mathrm{a}$ & $34.20 \mathrm{abc}$ & $49.86 c$ & 23.95ab & $25.26 a b$ \\
\hline 3-Methyl-butan-1-ol & $4.68 \mathrm{ab}$ & $5.55 \mathrm{ab}$ & $6.76 a b$ & $2.49 \mathrm{ab}$ & $1.14 \mathrm{a}$ & $5.20 \mathrm{ab}$ \\
\hline trans-2-Hexenal & 583.7abcdef & 610.5abcdefg & 826.9defg & 253.7abcd & $43.1 \mathrm{a}$ & 698.1bcdefg \\
\hline Pentan-1-ol & $0.27 a$ & $0.27 a$ & $1.85 \mathrm{abc}$ & $0.52 \mathrm{ab}$ & 1.39abc & $0.33 a$ \\
\hline cis-2-Penten-1-ol & $24.87 a$ & $8.78 a$ & $19.55 a$ & $31.38 a$ & $17.14 a$ & $13.10 a$ \\
\hline Hexan-1-ol & $17.42 \mathrm{a}$ & $36.32 a$ & $8.81 \mathrm{a}$ & $2.75 a$ & $2.53 a$ & $1.50 \mathrm{a}$ \\
\hline cis-3-Hexen-1-ol & $26.82 \mathrm{ab}$ & $38.41 \mathrm{abc}$ & $17.08 \mathrm{ab}$ & 61.04abc & $25.14 \mathrm{ab}$ & $2.61 \mathrm{a}$ \\
\hline trans-2-Hexen-1-ol & $26.95 a$ & $132.07 b$ & $12.54 a$ & $3.94 a$ & $1.88 \mathrm{a}$ & $8.48 a$ \\
\hline Acetic acid & $0.42 \mathrm{a}$ & $0.28 \mathrm{a}$ & $0.31 \mathrm{a}$ & $0.24 a$ & $0.46 a$ & $0.35 a$ \\
\hline Octan-1-ol & $0.63 a$ & $2.81 \mathrm{~b}$ & $0.40 a$ & $0.62 a$ & $0.53 a$ & $0.41 \mathrm{a}$ \\
\hline Total alcohols & $174.53 a$ & $258.81 a$ & $127.71 \mathrm{a}$ & $178.57 a$ & $112.25 a$ & $90.50 a$ \\
\hline Total aldehydes & 610.73abcd & 669.18abcd & $862.58 \mathrm{bcd}$ & $322.25 a b c$ & $67.33 a$ & 766.86abcd \\
\hline Total ketones & $20.19 a$ & 35.41 & 16.67 & 33.04 & 13.27 & 9.98 \\
\hline Total $C_{5}$ compounds & $83.43 \mathrm{ab}$ & $37.78 a$ & 68.93ab & $121.57 b$ & $51.82 \mathrm{ab}$ & $48.52 \mathrm{ab}$ \\
\hline Total $\mathrm{C}_{6}$ compounds & 673.60ab & 859.59ab & 890.37ab & 361.18ab & $87.32 a$ & 730.74ab \\
\hline Total $C_{6}$ from LA & $36.09 a$ & $78.61 \mathrm{a}$ & $33.85 a$ & $42.48 \mathrm{a}$ & $17.16 a$ & $21.51 \mathrm{a}$ \\
\hline Total $C_{6}$ from $\operatorname{Ln} A$ & 637.52abc & 780.98abc & $856.52 \mathrm{abc}$ & 318.70ab & $70.15 a$ & 709.23abc \\
\hline Total volatiles & $834.5 \mathrm{abc}$ & $976.5 b c$ & $1016.4 b c$ & 541.4ab & $205.6 a$ & $882.0 \mathrm{abc}$ \\
\hline Total phenols ${ }^{\mathrm{b}}$ & $326.2 \mathrm{f}$ & 245.6de & $128.4 \mathrm{bc}$ & $269.9 \mathrm{e}$ & $142.4 b c$ & $84.1 \mathrm{a}$ \\
\hline
\end{tabular}

LA, linoleic acid. LnA, linolenic acid.

a Rows: values with the same letters are not statistically different at $P=0.05$.

b From Tura et al. (2007).

separated by centrifugation at $2000 \mathrm{rpm}$. The oils were classed as "virgin" because the acidity value, the peroxide number, $K_{232}, K_{270}$ and $\Delta K$ of all oils were under the limits of the Commission Regulation (EC) no. 1989/2003. Due to alternate bearing, all oil samples were not available every year.

\subsection{Volatile compounds analysis}

The volatile composition was determined following the extraction procedure and GC analysis described in Angerosa et al. (1997).

\subsection{Sensorial analysis}

The sensory evaluation was carried out by the procedures described in the enclosure XII of the Commission Regulation (EC) no. $796 / 2002$, but modifying the sensorial profile sheet according to a parametric non-structured assessment based on many olfactory, gustatory and tactile descriptors. About $15-20 \mathrm{~mL}$ of oil were put in blue glasses warmed at $28-30^{\circ} \mathrm{C}$. Eight trained tasters from three different panel groups evaluated the sensory notes of all samples. Data was expressed in arbitrary units (A.U.). 'Green' notes include the following descriptors: 'lawn', 'leaf',

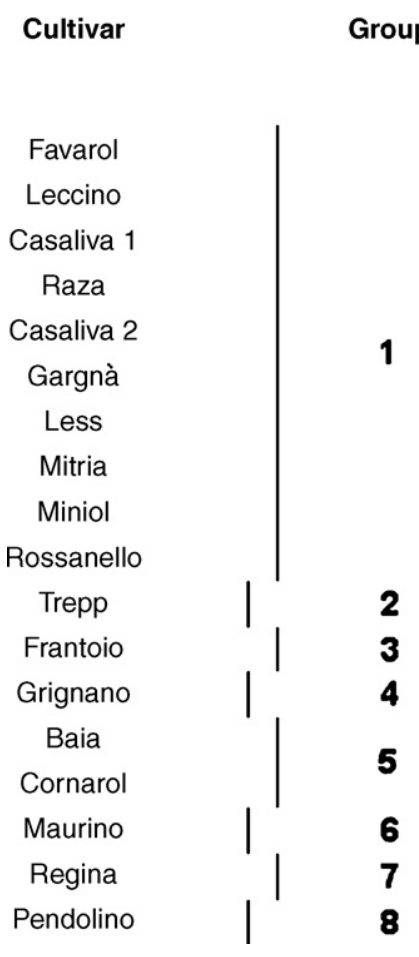
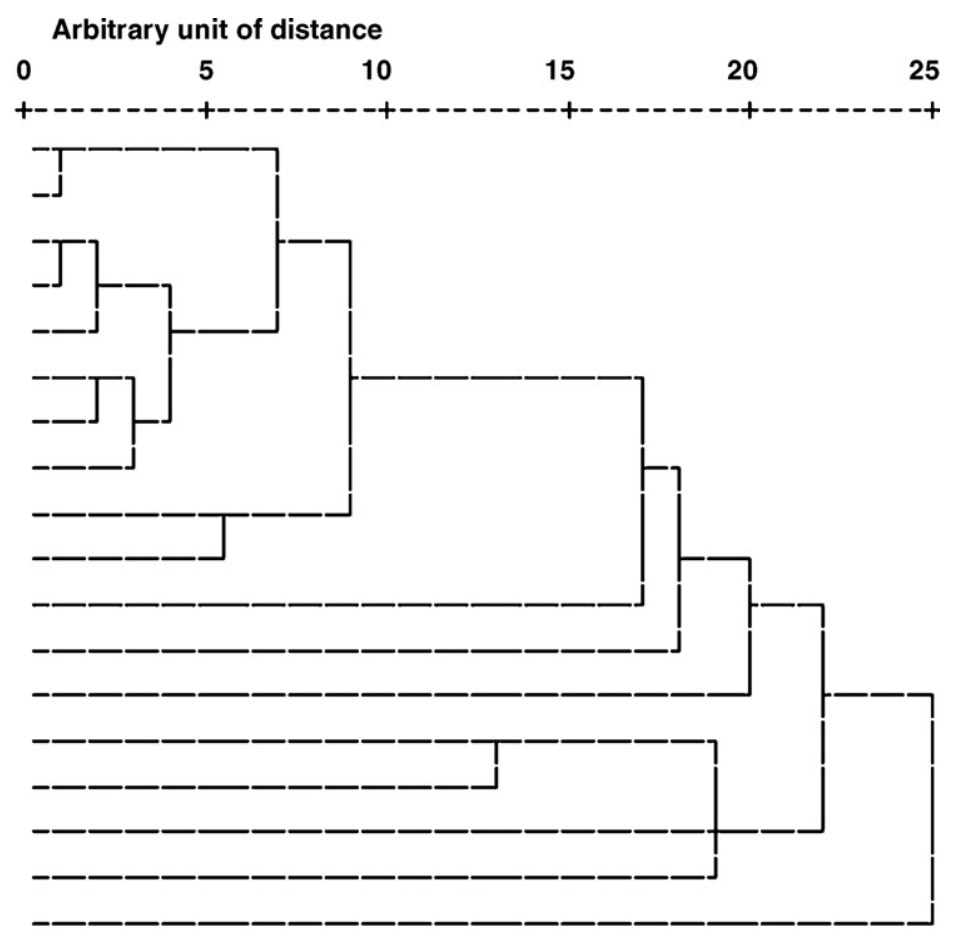

Fig. 1. Oil aromatic profiling: similarity groups dendrogram. 


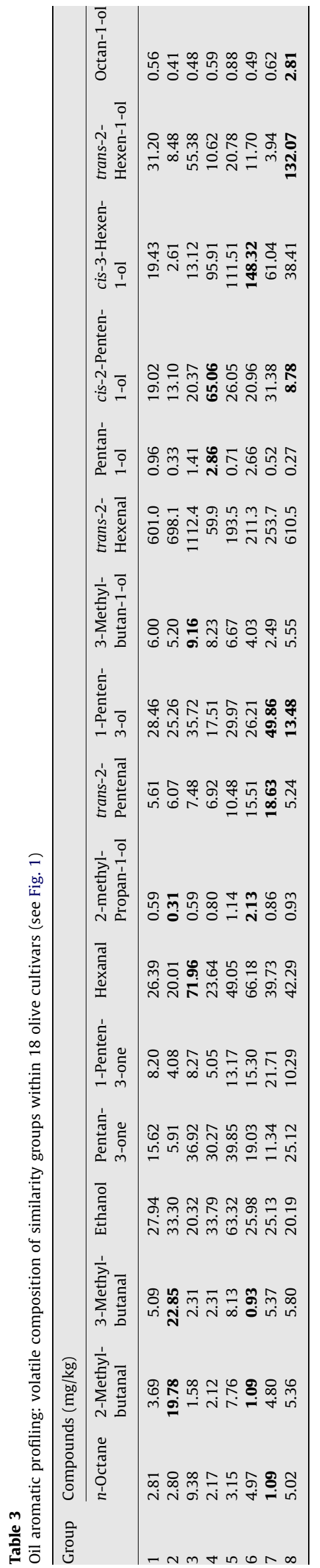

'artichoke', 'walnut' and 'hay'. 'Floral' notes include 'flowers' and 'butter' descriptors. 'Fruity' notes include 'olives', 'banana', 'tomato', 'almond' and 'apple' descriptors. 'Taste' notes include 'bitter', 'pungent' and 'astringency'. 'Satisfaction' is the overall hedonistic score considering all together the attributes of aroma, taste and flavor.

\subsection{Statistical analysis}

Normal distribution of chemical and physical variables was checked by the Kolmogorov-Smirnov test. When distribution was not normal, the standard deviation was not reported in summary tables. Outliers data, however, were not excluded in order to keep track of the peculiar composition of oils from diverse cultivar. In order to test the significance of the differences among chemical and sensory variables in relation to cultivars, data were processed by a general linear model which included it as sources of variability. Means were separated according to Duncan's multiple comparison test. The magnitude of variability in chemical oil composition and sensory notes, due to the cultivar, was quantified in terms of the expected components of the variance.

In order to group cultivars according to oil chemical composition and sensory evaluation, the four-year data was processed by cluster analysis via the square Euclidean distance and the average linkage between groups as clustering method. Results were represented with rooted dendrograms. A rooted dendrogram is a bidimensional graph representing the results of the classification of the single case into hierarchical groups. The level of each branching along the distance axe represents the distance between the groups, according to the adopted distance measure and clustering method. Data from panel test were normalized by panelist via normal score transformation: $z=(x-\mu) / \sigma . z$ represents an arbitrary unit that, for our data, ranged between -1 and +1 . To express sensory score with a positive scale, data were transformed according to the following linear function: $z_{1}=(z+1)^{*} 5$.

Relationships between the sensory attributes and the oil chemical composition were tested by simple linear regression analysis. Moreover, other multiple linear regression models based

Table 4

Oil sensorial profiling (3-4 years average): number of samples, value range, mean, standard deviation (expressed as arbitrary unites: A.U.) and expected variance component due to cultivar and its interactions

\begin{tabular}{lllllr}
\hline Descriptor & $N$ & Range (A.U.) & Mean (A.U.) & S.D. (A.U.) & Variance (\%) \\
\hline Lawn & 60 & $0.74-14.8$ & 5.56 & 2.71 & 9 \\
Leaf & 60 & $2.25-9.76$ & 5.34 & 2.04 & 12 \\
Olives & 60 & $0.27-11.0$ & 5.33 & 2.76 & 42 \\
Flowers & 60 & $4.04-12.9$ & 5.30 & n.r. & 75 \\
Banana & 60 & $2.97-14.9$ & 5.26 & n.r. & 82 \\
Tomato & 60 & $3.23-10.9$ & 5.39 & 2.09 & 32 \\
Almond & 60 & $2.98-11.9$ & 5.27 & n.r. & 1 \\
Artichoke & 60 & $2.70-12.3$ & 5.42 & n.r. & 9 \\
Apple & 60 & $4.39-10.4$ & 5.16 & n.r. & 77 \\
Walnut & 60 & $2.00-14.5$ & 5.37 & n.r. & 79 \\
Hay & 60 & $3.11-11.9$ & 5.28 & n.r. & 94 \\
Butter & 60 & $3.71-10.8$ & 5.19 & n.r. & 63 \\
Bitter & 60 & $0.95-16.3$ & 5.76 & 2.62 & 13 \\
Sweet & 60 & $1.11-10.6$ & 5.08 & 1.94 & 69 \\
Pungent & 60 & $1.27-9.5$ & 5.22 & 2.10 & 10 \\
Astringency & 60 & $2.67-10.5$ & 5.28 & n.r. & 0 \\
Green notes & 60 & $16.6-39.7$ & 27.0 & 4.85 & 31 \\
Floral notes & 60 & $7.77-21.8$ & 10.5 & n.r. & 81 \\
Fruity notes & 60 & $18.5-36.0$ & 26.4 & 4.41 & 68 \\
Taste notes & 60 & $6.72-26.4$ & 16.3 & 4.73 & 1 \\
Satisfaction & 60 & $0.00-24.2$ & 10.2 & 6.81 & 4 \\
\hline
\end{tabular}

n.r., not reported because frequency distributions were not normal according to Kolmogorov-Smirnov test $(P=0.05)$. 
Table 5

Oil sensorial profiling: comparison among 18 cultivars (within brackets: number of cropping years)

\begin{tabular}{|c|c|c|c|c|c|c|}
\hline Descriptor (A.U.) & Baia (3) & Casaliva 1 (4) & Casaliva 2 (4) & Cornarol (3) & Favarol (4) & Frantoio (4) \\
\hline Lawn & $4.77 \mathrm{ab}^{\mathrm{a}}$ & $6.15 b c$ & $6.14 \mathrm{bc}$ & $8.81 \mathrm{~d}$ & $4.70 \mathrm{ab}$ & $5.51 \mathrm{abc}$ \\
\hline Leaf & $6.91 d$ & 7.51d & 5.60abcd & 5.71abcd & 5.96abcd & $5.85 \mathrm{abcd}$ \\
\hline Olives & $6.74 \mathrm{ef}$ & 6.12cdef & $5.93 c d e f$ & $7.02 f$ & $5.59 \mathrm{bcdef}$ & $6.88 \mathrm{ef}$ \\
\hline Flowers & $5.85 \mathrm{ab}$ & $5.21 \mathrm{ab}$ & $4.28 \mathrm{a}$ & $4.57 \mathrm{a}$ & $5.75 a b$ & $4.66 a$ \\
\hline Banana & 4.06a & $4.53 a$ & $5.59 \mathrm{ab}$ & $4.94 a$ & $4.49 a$ & $5.63 a b$ \\
\hline Tomato & $4.26 \mathrm{abc}$ & $4.54 \mathrm{abc}$ & $6.14 c d$ & $9.08 \mathrm{e}$ & $7.15 d$ & $4.15 \mathrm{abc}$ \\
\hline Almond & $3.96 a$ & $5.41 \mathrm{ab}$ & $5.36 \mathrm{ab}$ & $4.88 \mathrm{ab}$ & $4.75 \mathrm{ab}$ & $5.47 \mathrm{ab}$ \\
\hline Artichoke & $4.59 \mathrm{abc}$ & $4.98 \mathrm{abc}$ & $4.21 \mathrm{abc}$ & 7.65de & 7.46de & $3.60 a b$ \\
\hline Apple & $7.11 \mathrm{~cd}$ & $4.57 a$ & $5.00 \mathrm{ab}$ & 5.29ab & 5.13ab & $5.12 \mathrm{ab}$ \\
\hline Walnut & $6.17 \mathrm{bcd}$ & $5.72 \mathrm{abc}$ & 4.79ab & 5.26abc & $5.33 \mathrm{abc}$ & $4.53 \mathrm{ab}$ \\
\hline Hay & $3.82 \mathrm{a}$ & $6.73 b c$ & $4.31 \mathrm{a}$ & $4.61 \mathrm{a}$ & $5.14 \mathrm{ab}$ & $5.00 \mathrm{ab}$ \\
\hline Butter & $4.25 \mathrm{ab}$ & 4.64ab & 4.33ab & 4.65ab & $4.62 \mathrm{ab}$ & $6.98 \mathrm{~d}$ \\
\hline Bitter & $8.26 \mathrm{e}$ & $5.62 \mathrm{bcd}$ & $5.34 \mathrm{bcd}$ & 4.79abcd & $6.10 \mathrm{cde}$ & 5.89bcde \\
\hline Sweet & 4.73abc & $5.09 \mathrm{abc}$ & 5.61abcd & $4.98 \mathrm{abc}$ & $3.87 a$ & 4.44abc \\
\hline Pungent & $8.48 f$ & $6.20 \mathrm{e}$ & 4.47abcde & $5.63 \mathrm{cde}$ & 5.32bcde & $6.59 \mathrm{ef}$ \\
\hline Astringency & $4.09 a$ & 5.29abcd & $4.83 a b c d$ & $5.32 \mathrm{abcd}$ & $6.09 \mathrm{cde}$ & $4.98 \mathrm{abcd}$ \\
\hline 'Green' notes & 26.26abc & $31.09 \mathrm{bc}$ & $25.05 \mathrm{abc}$ & $32.05 \mathrm{c}$ & $28.58 \mathrm{abc}$ & 24.49abc \\
\hline 'Floral' notes & 10.11abcde & 9.85abcd & $8.61 \mathrm{ab}$ & 9.23abc & $10.36 \mathrm{bcdef}$ & $11.64 \mathrm{efg}$ \\
\hline 'Fruity' notes & $26.13 \mathrm{bcd}$ & $25.16 a b c$ & 28.02bcde & $31.21 \mathrm{de}$ & 27.11 bcde & 27.25bcde \\
\hline 'Taste' notes & $20.84 a$ & 17.11a & $14.64 a$ & $15.74 a$ & $17.51 \mathrm{a}$ & $17.45 a$ \\
\hline Satisfaction & $9.09 \mathrm{bcdef}$ & 11.95defgh & 17.30h & $15.04 \mathrm{gh}$ & $6.30 \mathrm{abc}$ & 7.40abcde \\
\hline Descriptor (A.U.) & Gargnà (3) & Grignano (2) & Leccino (4) & Less (3) & Maurino (3) & Miniol (4) \\
\hline Lawn & $5.22 \mathrm{abc}$ & 3.16a & $3.92 \mathrm{ab}$ & $3.17 \mathrm{a}$ & 5.17abc & $5.41 \mathrm{abc}$ \\
\hline Leaf & 5.06abcd & $3.40 a$ & 5.58abcd & 4.29abc & 3.79ab & $6.22 \mathrm{bcd}$ \\
\hline Olives & $6.36 \mathrm{cdef}$ & $2.96 \mathrm{ab}$ & 3.72abcd & $3.61 \mathrm{abc}$ & $5.99 \mathrm{cdef}$ & 5.37bcdef \\
\hline Flowers & $4.21 \mathrm{a}$ & $8.18 \mathrm{c}$ & $5.89 a b$ & $5.64 \mathrm{ab}$ & $4.56 a$ & $4.86 a$ \\
\hline Banana & $6.98 \mathrm{bc}$ & $6.96 b c$ & $4.96 a$ & $5.02 a$ & $7.29 b c$ & $7.52 \mathrm{c}$ \\
\hline Tomato & $4.76 \mathrm{abc}$ & $4.01 \mathrm{abc}$ & $4.67 \mathrm{abc}$ & $4.25 \mathrm{abc}$ & $5.87 \mathrm{bcd}$ & $3.51 \mathrm{a}$ \\
\hline Almond & $6.22 \mathrm{bc}$ & 5.16ab & 5.90abc & 7.67c & 4.58ab & $4.51 \mathrm{ab}$ \\
\hline Artichoke & $3.28 \mathrm{a}$ & $3.84 \mathrm{abc}$ & $5.20 \mathrm{abc}$ & 4.32abc & $3.23 a$ & 7.32de \\
\hline Apple & 4.83ab & $4.51 \mathrm{a}$ & $4.57 a$ & $6.31 b c$ & $7.90 \mathrm{~d}$ & $4.57 a$ \\
\hline Walnut & $7.20 \mathrm{~cd}$ & $6.01 \mathrm{bc}$ & $4.77 a b$ & $5.23 \mathrm{abc}$ & $5.37 \mathrm{abc}$ & $5.76 \mathrm{abc}$ \\
\hline Hay & $5.05 \mathrm{ab}$ & $4.27 a$ & $7.13 c$ & $5.73 a b c$ & $5.68 \mathrm{abc}$ & 5.19abc \\
\hline Butter & 5.62abcd & $5.95 \mathrm{bcd}$ & $6.57 c d$ & $5.06 a b c$ & 5.27abcd & $4.78 \mathrm{ab}$ \\
\hline Bitter & 7.33de & 2.69a & 4.13abc & $5.46 \mathrm{bcd}$ & $5.45 \mathrm{bcd}$ & 6.85de \\
\hline Sweet & 4.13ab & $7.47 d$ & $6.64 \mathrm{~cd}$ & $4.59 \mathrm{abc}$ & $4.84 \mathrm{abc}$ & 5.71abcd \\
\hline Pungent & 4.88abcde & 3.07ab & 3.72abc & 6.13de & 3.80abcd & 4.42abcde \\
\hline Astringency & $5.02 \mathrm{abcd}$ & 4.32ab & $4.53 \mathrm{abc}$ & 4.74abc & 6.39de & 7.11e \\
\hline 'Green' notes & $25.82 \mathrm{abc}$ & 20.69a & $26.60 \mathrm{abc}$ & 22.74ab & $23.24 a b$ & $29.90 b c$ \\
\hline 'Floral' notes & 9.83abcd & 14.13h & $12.46 \mathrm{gh}$ & 10.70cdefg & $9.83 \mathrm{abcd}$ & 9.64abcd \\
\hline 'Fruity' notes & 29.14 cde & 23.59ab & $23.82 \mathrm{ab}$ & $26.86 \mathrm{bcde}$ & $31.63 e$ & $25.48 \mathrm{abc}$ \\
\hline 'Taste' notes & $17.23 \mathrm{a}$ & $10.09 a$ & $12.37 \mathrm{a}$ & $16.33 a$ & $15.65 a$ & $18.38 \mathrm{a}$ \\
\hline Satisfaction & $13.40 \mathrm{fgh}$ & 6.98abcd & 7.20abcde & 11.27cdefg & 6.82abcd & 11.97defgh \\
\hline Descriptor (A.U.) & Mitria (4) & Pendolino (3) & Raza (3) & Regina (3) & Rossanello (3) & Trepp (3) \\
\hline Lawn & $7.42 \mathrm{~cd}$ & $3.32 \mathrm{a}$ & $5.70 \mathrm{bc}$ & $9.26 \mathrm{~d}$ & $4.83 \mathrm{ab}$ & $5.90 \mathrm{bc}$ \\
\hline Leaf & $6.49 \mathrm{~cd}$ & $3.43 a$ & 5.04abcd & 5.71abcd & $3.82 \mathrm{ab}$ & 4.13abc \\
\hline Olives & $5.95 \mathrm{cdef}$ & $1.86 a$ & $6.47 \mathrm{def}$ & 5.43 bcdef & 4.67bcdef & 4.16abcde \\
\hline Flowers & $5.55 \mathrm{ab}$ & $4.97 a$ & $4.25 a$ & $6.59 \mathrm{~b}$ & $5.47 \mathrm{ab}$ & $5.42 \mathrm{ab}$ \\
\hline Banana & $4.19 \mathrm{a}$ & $4.69 a$ & $4.44 a$ & $4.41 \mathrm{a}$ & 5.63ab & $4.61 \mathrm{a}$ \\
\hline Tomato & $4.65 a b c$ & 3.92ab & $6.02 \mathrm{bcd}$ & $6.88 \mathrm{~d}$ & 7.54de & $6.04 \mathrm{bcd}$ \\
\hline Almond & $5.16 a b$ & $5.88 \mathrm{abc}$ & $3.99 a$ & $4.07 a$ & $4.45 \mathrm{ab}$ & $6.51 \mathrm{bc}$ \\
\hline Artichoke & $5.56 \mathrm{bcd}$ & $4.86 \mathrm{abc}$ & $7.74 \mathrm{e}$ & $5.11 \mathrm{abc}$ & $5.91 \mathrm{cde}$ & 7.65de \\
\hline Apple & 4.99ab & $4.63 a$ & $5.03 a b$ & $5.49 \mathrm{ab}$ & $4.90 \mathrm{ab}$ & $4.55 a$ \\
\hline Walnut & $3.75 a$ & $8.00 \mathrm{~d}$ & $4.48 \mathrm{ab}$ & $4.57 a b$ & $6.32 \mathrm{bcd}$ & $6.28 \mathrm{bcd}$ \\
\hline Hay & $5.84 \mathrm{abc}$ & $4.41 \mathrm{a}$ & $5.75 a b c$ & $3.95 a$ & $4.51 \mathrm{a}$ & 5.79abc \\
\hline Butter & $4.82 \mathrm{abc}$ & $6.05 \mathrm{bcd}$ & $4.06 a$ & 5.39abcd & $4.59 \mathrm{ab}$ & $5.94 \mathrm{bcd}$ \\
\hline Bitter & $6.02 \mathrm{cde}$ & $3.32 \mathrm{ab}$ & $5.15 \mathrm{bcd}$ & 6.34cde & 7.07de & $6.58 \mathrm{cde}$ \\
\hline Sweet & $4.16 a b$ & 5.30abcd & 6.15abcd & 3.75a & $4.89 \mathrm{abc}$ & $6.51 \mathrm{bcd}$ \\
\hline Pungent & 5.09abcde & $2.82 a$ & $6.30 \mathrm{ef}$ & $6.39 \mathrm{ef}$ & $6.45 \mathrm{ef}$ & 5.51cde \\
\hline Astringency & $5.28 \mathrm{abcd}$ & $4.10 \mathrm{a}$ & $4.81 \mathrm{abcd}$ & 5.84 bcde & 5.79bcde & 5.23abcd \\
\hline 'Green' notes & $29.06 a b c$ & $24.02 \mathrm{abc}$ & 28.72abc & 28.59abc & $25.40 \mathrm{abc}$ & $29.76 b c$ \\
\hline 'Floral' notes & 10.37bcdef & 11.02defg & 8.31a & 11.98fgh & 10.06abcde & $11.36 \mathrm{cdef}$ \\
\hline 'Fruity' notes & 24.93abc & $20.97 a$ & 25.94abcd & $26.28 \mathrm{bcd}$ & 27.19bcde & $25.86 a b c$ \\
\hline 'Taste' notes & $16.38 a$ & $10.24 a$ & $16.26 a$ & $18.56 a$ & 19.31a & $17.32 \mathrm{a}$ \\
\hline Satisfaction & $5.24 \mathrm{ab}$ & 2.88a & $15.80 \mathrm{gh}$ & 12.61 efgh & 10.72bcdefg & 8.76bcdef \\
\hline
\end{tabular}

a Rows: values with the same letters are not statistically different at $P=0.05$. 
on the different technique (i.e. enter, stepwise forward and backward elimination method) were assayed, although without any significant result.

Data were processed by an SPSS statistical package (version 14.0 for Window-SPSS Inc., Chicago, Illinois, 2006).

Only the average data were reported, in order to highlight the cultivar patterns, independently from season influences.

\section{Results}

\subsection{Aromatic profiling}

For a better understanding of the aromatic profile of each cultivar, the total content of volatile compounds with chemical affinity or similar biosynthetic pathway was taken in account (Table 1). The total volatile compounds ranged from $62.8 \mathrm{mg} / \mathrm{kg}$ ('Rossanello' in 1998) to $2184.2 \mathrm{mg} / \mathrm{kg}$ ('Casaliva 1' in 2001). Among the major compounds, total alcohols ranged from $52.4 \mathrm{mg} /$ $\mathrm{kg}$ to $541.3 \mathrm{mg} / \mathrm{kg}$, including ethanol, which is a precursor of several aroma compounds; total aldehydes ranged from 2.55 to 1926.4, including 2- and 3-methyl-butal that are not originated from lipoxygenase pathway (LOX); total ketones, ranged from 3.63 to 127.2. Considering the volatiles from LOX, the total amount of $C_{5}$ and $\mathrm{C}_{6}$ compounds from linolenic acid $(\operatorname{LnA})$ oxidation ranged from 4.54 to 198.8 and from 18.3 to 1942.5 , respectively; and the total amount of $\mathrm{C}_{6}$ compounds, from linoleic acid (LA) oxidation ranged from 5.20 to 170.9 .

The volatiles composition of the oil head-space for each cultivar is reported in Table 2. Differences in ethyl acetate, hexan-1-ol, acetic acid, total alcohols and ketones, and the total amount of $\mathrm{C}_{6}$ compounds (from LOX of LA) were not significant in distinguishing among cultivars. Some cultivars showed a peculiar aromatic profile, e.g. 'Baia', high content of ethanol; 'Casaliva 1', high trans-2hexenal, total aldehydes and $C_{6}$ volatiles and low ethanol; 'Cornarol', high pentan-3-one; 'Favarol', low trans-2-pentanal; 'Frantoio', high n-octane, hexanal, 3-methyl-butanal-1-ol and total volatiles; 'Grignano', high pentan-1-ol and cis-2-penten-1-ol; 'Maurino', low 2- and 3-methyl-butanal, and high 2-methylpropan-1-ol and cis-3-hexen-1-ol; 'Miniol', low hexanal and trans2-hexenal; 'Mitria', high total phenols; 'Pendolino', low 1-penten3-ol, cis-2-penten-1-ol and total $C_{5}$ compounds, and high trans-2hexen-1-ol and octan-1-ol; 'Raza', low octan-1-ol; 'Regina', low noctane, and high 1-penten-3-one, trans-2-pentenal, 1-penten-3-ol and total $C_{5}$ compounds; 'Rossanello', low 3-methyl-butan-1-ol, trans-2-hexen-1-ol, total aldehydes, total $\mathrm{C}_{6}$ compounds and total volatiles; 'Trepp', high 2 and 3-methyl-butanal, and low pentan-3one, 1-penten-3-one, 2-methyl-propan-1-ol, cis-3-hexen-1-ol and total phenols.

According to unique aromatic profile eight affinity groups were built (Fig. 1, Table 3). Group 1 featured by a medium aromatic

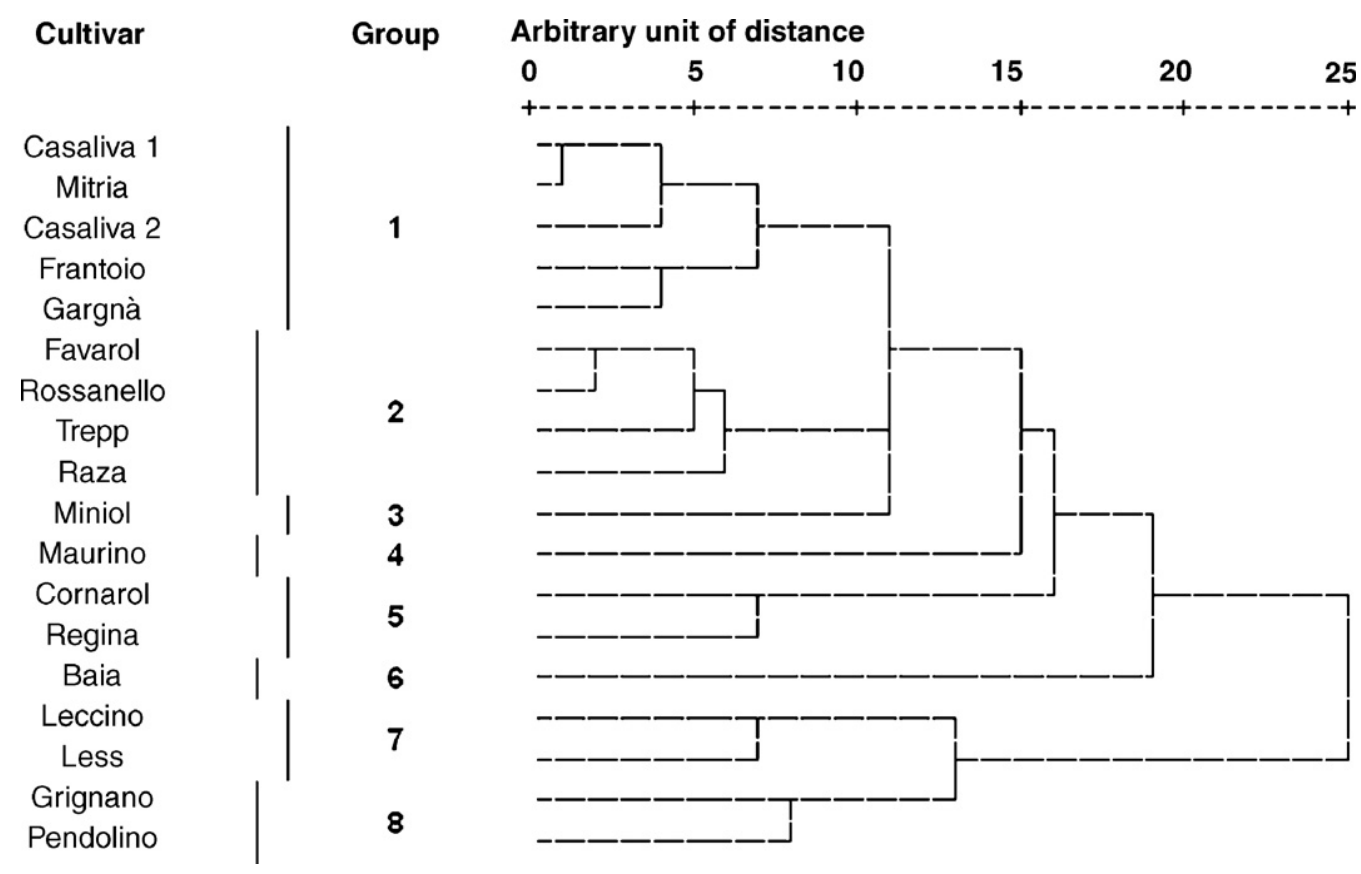

Fig. 2. Oil sensorial profiling: similarity groups dendrogram.

Table 6

Oil sensorial profiling: sensory notes of similarity groups within 18 olive cultivars (see Fig. 2)

\begin{tabular}{|c|c|c|c|c|c|c|c|c|c|c|c|c|c|c|c|c|}
\hline \multirow[t]{2}{*}{ Group } & \multicolumn{16}{|c|}{ Descriptor (A.U.) } \\
\hline & Lawn & Leaf & Olives & Flowers & Banana & Tomato & Almond & Artichoke & Apple & Walnut & Hay & Butter & Bitter & Sweet & Pungent & Astringency \\
\hline 1 & 6.09 & 6.10 & 6.25 & 4.78 & 5.38 & 4.85 & 5.52 & 4.33 & 4.90 & 5.20 & 5.39 & 5.28 & 6.04 & 4.69 & 5.44 & 5.08 \\
\hline 2 & 5.28 & 4.74 & 5.22 & 5.22 & 4.79 & 6.69 & 4.92 & 7.19 & 4.90 & 5.60 & 5.30 & 4.80 & 6.23 & 5.36 & 5.90 & 5.48 \\
\hline 3 & 5.41 & 6.22 & 5.37 & 4.86 & 7.52 & 3.51 & 4.51 & 7.32 & 4.57 & 5.76 & 5.19 & 4.78 & 6.85 & 5.71 & 4.42 & 7.11 \\
\hline 4 & 5.17 & 3.79 & 5.99 & 4.56 & 7.29 & 5.87 & 4.58 & 3.23 & 7.90 & 5.37 & 5.68 & 5.27 & 5.45 & 4.84 & 3.80 & 6.39 \\
\hline 5 & 9.03 & 5.71 & 6.22 & 5.58 & 4.68 & 7.98 & 4.47 & 6.38 & 5.39 & 4.91 & 4.28 & 5.02 & 5.56 & 4.36 & 6.01 & 5.58 \\
\hline 6 & 4.77 & 6.91 & 6.74 & 5.85 & 4.06 & 4.26 & 3.96 & 4.59 & 7.11 & 6.17 & 3.82 & 4.25 & 8.26 & 4.73 & 8.48 & 4.09 \\
\hline 7 & 3.54 & 4.94 & 3.67 & 5.76 & 4.99 & 4.46 & 6.79 & 4.76 & 5.44 & 5.00 & 6.43 & 5.82 & 4.80 & 5.62 & 4.92 & 4.63 \\
\hline 8 & 3.24 & 3.41 & 2.41 & 6.57 & 5.83 & 3.96 & 5.52 & 4.35 & 4.57 & 7.01 & 4.34 & 6.00 & 3.01 & 6.39 & 2.95 & 4.21 \\
\hline
\end{tabular}


composition ('Favarol', 'Leccino', 'Casaliva 1', 'Raza', 'Casaliva 2', 'Gargnà', 'Less', 'Mitria', 'Miniol' and 'Rossanello') and group 5 by a high content of ethanol and pentan-3-one ('Baia' and 'Cornarol'). The following cultivars showed a unique profile: 'Trepp', 'Frantoio', 'Grignano', 'Maurino', 'Regina' and 'Pendolino'.

\subsection{Sensorial profiling}

The sensorial traits of the oils are shown in Table 4. 'Green' notes ranged from 16.6 A.U. to 39.7 A.U.; 'floral' notes from 7.77 to 21.8; 'fruity' notes, from 18.5 to 36.0 ; 'taste' notes, from 6.72 to 26.4; 'satisfaction', from 0 ('Maurino' in 1999) to 24.2 ('Casaliva 2 ' in 2000).

The monovarietal oils were discriminated by their sensorial traits, except for the 'taste' notes (Table 5). 'Baia' showed low notes of 'banana', 'almond', 'hay' and 'astringency', and high of 'bitter' and 'pungent'; 'Casaliva 1', low in 'leaf'; 'Casaliva 2', the highest for 'satisfaction'; 'Cornarol', high in 'olives', 'tomato' and 'green' notes; 'Frantoio', high in 'butter'; 'Gargnà', low in 'flowers'; 'Grignano', low in 'lawn', 'leaf', 'apple', 'bitter' and 'green' notes, and high in 'flowers', 'sweet' and 'floral' notes; 'Leccino', high in 'hay'; 'Less', high in 'almond'; 'Maurino', low in 'artichoke', and high in 'apple' and 'fruity' notes; 'Miniol', high in 'banana' and 'astringency', and low in 'tomato'; 'Mitria', low in 'walnut'; 'Pendolino', low in 'olives', 'pungent', 'fruity' notes and 'satisfaction', and high in 'walnut'; 'Raza', high in 'artichoke', and low in 'butter' and 'floral' notes; 'Regina', high in 'lawn' and low in 'sweet'.

When grouping cultivars according to their sensorial profile, eight affinity groups with a characteristic sensorial profile were distinguished (Fig. 2, Table 6). Group 1 showing a low-medium content of sensory notes ('Casaliva 1', 'Mitria', 'Casaliva 2', 'Frantoio' and 'Gargnà'); group 2, with a high-medium sensorial profile ('Favarol, 'Rossanello', 'Trepp' and 'Raza'); group 5, with high notes of 'lawn' and 'tomato', and low in 'walnut' and 'sweet' ('Cornarol' and 'Regina'); group 7, with high notes of 'almond' and 'hay' ('Leccino' and 'Less'); group 8, with low notes of 'lawn', 'leaf', 'olives', 'bitter', 'pungent', and high in 'flowers', 'walnut', 'butter' and 'sweet' ('Grignano' and 'Pendolino'). 'Miniol', Maurino' and 'Baia' were not grouped due to their peculiar profiles.

\section{Discussion}

The range of aromatic and sensorial attributes of the oils from the 18 assessed cultivars was in accordance with whose reported in the available literature (Dhifi et al., 2005; Luna et al., 2006; Berlioz et al., 2006; Haddada et al., 2007; Pedò et al., 2002, 2003). The variability of volatile compounds and sensory notes varied largely as a consequence of cultivar, year and their interactions, as already shown in previous works (Tura et al., in press, 2005). It was pointed out that olives ripening under higher heat courses produced oils containing more volatiles and phenols than the ones made from olives with similar maturity index. The variability of the following parameters depends mainly on cultivar: ethanol, 2-methyl-propan-1-ol, pentan-1-ol, cis-2-penten-1-ol, cis-3hexen-1-ol and octan-1-ol, for the chemical compounds; 'flowers', 'banana', 'apple', 'walnut', 'hay', 'butter', 'sweet', 'floral' and 'fruity' notes for the sensorial attributes.

\subsection{Cultivar characteristics}

Some oils from local cultivars showed a peculiar aromatic and sensorial profile. 'Casaliva 1' has a flavor of 'leaf' for its high content of trans-2-hexenal, total aldehydes and many $C_{6}$ compounds. 'Cornarol' features flavor of 'olives', 'tomato' and 'green' notes for high pentan-3-one content. 'Grignano' has a mild flavor for its 'flowers', 'sweet' and 'floral' notes, and high content of pentan-1-ol and cis-2-penten-1-ol. 'Maurino' has flavor of 'apple' and 'fruity' notes probably due to a high content of 2-methyl-propan-1-ol and cis-3-hexen-1-ol. 'Pendolino' has flavor of 'walnut' most likely explained by a high content of trans-2-hexen-1-ol. 'Regina' has flavor of 'lawn' probably for its high content of pentan-3-one, trans2-pentenal, 1-penten-3-ol and $C_{5}$ compounds.

\subsection{Cultivar similarity}

It is important to note that some cultivars, with a similar aromatic content, showed also an analogous sensorial profile. 'Favarol', 'Casaliva 1', 'Raza', 'Casaliva 2', 'Gargnà', 'Mitria', 'Miniol', and 'Rossanello' resulted in the same aromatic group, characterized by an average volatiles content, and in three close affinity groups (1-3), with a middle sensorial profile. Moreover, 'Maurino' was always isolated, showing peculiar profiles. Some of these similarities between volatiles and sensory notes confirmed previous findings (Pedò et al., 2002; Tura et al., 2002), supplying them with further details.

\subsection{Aromatic compounds and sensory notes correlations}

After assessing the chemical and sensorial characteristics of monovarietal olive oil and their similarity, some considerations can be drawn about correlations between volatile compounds and sensorial attributes (Table 7). The 'green' notes of 'lawn' and 'leaf' were correlated with hexanal, trans-2-pentanal, 1-penten-3-ol and total phenols; the 'fruity' notes of 'olives', 'banana', 'almond' and

Table 7

Linear correlations among oils sensory notes (descriptors) and aromatic chemical compounds

\begin{tabular}{|c|c|c|c|}
\hline Descriptor & Compound & $r$ & sig. \\
\hline Lawn & $\begin{array}{l}\text { Hexanal } \\
\text { trans-2-Pentenal } \\
\text { 1-Penten-3-ol }\end{array}$ & $\begin{array}{l}0.27 \\
0.39 \\
0.43\end{array}$ & $\begin{array}{l}0.04 \\
0.00 \\
0.00\end{array}$ \\
\hline Olives & $\begin{array}{l}\text { Ethyl acetate } \\
\text { trans-2-Pentenal } \\
\text { 1-Penten-3-ol }\end{array}$ & $\begin{array}{l}0.26 \\
0.27 \\
0.37\end{array}$ & $\begin{array}{l}0.05 \\
0.04 \\
0.00\end{array}$ \\
\hline Banana & $\begin{array}{l}\text { Pentan-1-ol } \\
\text { cis-2-Penten-1-ol } \\
\text { cis-3-Hexen-1-ol }\end{array}$ & $\begin{array}{l}0.25 \\
0.42 \\
0.26\end{array}$ & $\begin{array}{l}0.06 \\
0.00 \\
0.05\end{array}$ \\
\hline Almond & $\begin{array}{l}\text { Pentan-3-one } \\
\text { trans-2-Hexen-1-ol } \\
\text { Octan-1-ol }\end{array}$ & $\begin{array}{l}0.28 \\
0.33 \\
0.25\end{array}$ & $\begin{array}{l}0.03 \\
0.01 \\
0.06\end{array}$ \\
\hline Apple & $\begin{array}{l}\text { 2-Methyl-propan-1-ol } \\
\text { cis-3-Hexen-1-ol } \\
\text { Ethanol }\end{array}$ & $\begin{array}{l}0.42 \\
0.36 \\
0.34\end{array}$ & $\begin{array}{l}0.00 \\
0.01 \\
0.01\end{array}$ \\
\hline Leaf & $\begin{array}{l}\text { 1-Penten-3-ol } \\
\text { Total phenols }\end{array}$ & $\begin{array}{l}0.30 \\
0.28\end{array}$ & $\begin{array}{l}0.02 \\
0.03\end{array}$ \\
\hline Butter & $\begin{array}{l}\text { 3-Methyl-butan-1-ol } \\
\text { n-Octane }\end{array}$ & $\begin{array}{l}0.27 \\
0.29\end{array}$ & $\begin{array}{l}0.04 \\
0.03\end{array}$ \\
\hline Sweet & $\begin{array}{l}\text { 2-Methyl-butanal } \\
\text { 3-Methyl-butanal } \\
\text { Pentan-3-one } \\
\text { Pentan-1-ol } \\
\text { cis-2-Penten-1-ol }\end{array}$ & $\begin{array}{l}0.34 \\
0.37 \\
0.31 \\
0.25 \\
0.36\end{array}$ & $\begin{array}{l}0.01 \\
0.00 \\
0.02 \\
0.06 \\
0.01\end{array}$ \\
\hline Pungent & $\begin{array}{l}\text { Ethyl acetate } \\
\text { Ethanol } \\
\text { 1-Penten-3-ol } \\
\text { Pentan-1-ol }\end{array}$ & $\begin{array}{l}0.30 \\
0.33 \\
0.38 \\
0.25\end{array}$ & $\begin{array}{l}0.02 \\
0.01 \\
0.00 \\
0.05\end{array}$ \\
\hline Astringency & Ethyl acetate & 0.52 & 0.00 \\
\hline Satisfaction & 1-Penten-3-ol & 0.32 & 0.01 \\
\hline
\end{tabular}


Table 8

Relationships among sensory notes and aromatic chemical compounds in olive oils found by several authors (Burdock, 2002; Kalua et al., 2007; Angerosa et al., 2004)

\begin{tabular}{|c|c|}
\hline Descriptor & Chemical compound \\
\hline Green notes & $\begin{array}{l}\text { 2-Methyl propan-1-ol; cis-2-penten-1-ol; 2-hexen-1-ol; 3-hexen-1-ol } \\
\text { 2-Pentenal; hexanal; 2-hexenal; 3-hexenal; trans-2-octenal } \\
\text { Pentan-3-one; 4-methyl-pentan-2-one; nonan-2-one } \\
\text { Methyl acetate; buthyl acetate; hexyl acetate; 3-hexenyl acetate; ethyl propionate; methyl decanoate } \\
\text { 1-Octene; ethyl furano }\end{array}$ \\
\hline Olive fruity & $\begin{array}{l}\text { Pentan-1-ol; 4-methyl-1-penten-3-ol; hexan-1-ol } \\
\text { 3-methyl-butanal; 2-methyl-2-butenal; cis-2-pentenal; cis-2-hexenal; trans-3-hexenal; 2,4-hexadienal } \\
\text { Butan-2-one; eptan-2-one; 6-methyl-5-epten-2-one; octan-2-one; nonan-2-one } \\
\text { 3-Methyl-butyl acetate; hexyl acetate; 3-hexenyl acetate; 2-methyl-buthyl propionate; ethyl-methyl } \\
\text { butirrate; 3,4-dimethyl-3-pentenyl furano; ethyl cyclohexanoate; methyl benzene; ethyl benzene }\end{array}$ \\
\hline Apple & $\begin{array}{l}\text { trans-2-Pentenal; hexanal; 3-hexenal } \\
\text { Butan-2-one; nonan-2-one; ethyl propinate; 2-methyl-buthyl propionate }\end{array}$ \\
\hline $\begin{array}{l}\text { Flowers } \\
\text { Artichoke } \\
\text { Almond } \\
\text { Hay } \\
\text { Banana }\end{array}$ & $\begin{array}{l}\text { trans-3-Hexenal } \\
\text { trans-3-Hexenal } \\
\text { 2-Hexenal } \\
\text { 2-Methyl-4-pentenal } \\
\text { cis-2-Penten-1-ol; cis-3-hexen-1-ol; 3-methyl-buthyl acetate; 3-hexenyl acetate }\end{array}$ \\
\hline Sweet & $\begin{array}{l}\text { 4-Methyl-1-penten-3-ol; 3-methyl-butanal; hexanal } \\
\text { Pentan-3-one; 1-penten-3-one; 4-methyl-pentan-2-one; nonan-2-one } \\
\text { Ethyl acetate; buthyl acetate; hexyl acetate; ethyl propinate; ethyl furano }\end{array}$ \\
\hline Bitter & $\begin{array}{l}\text { 2-Methyl-3-buten-1-ol; trans-3-hexen-1-ol; 2-methyl-4-pentenal; 2-hexenal } \\
\text { 6-Methyl-5-hepten-2-one; 3-methyl-buthyl acetate; 2-methyl-buthyl propionate; methyl decanoate } \\
\text { Dodecene; tridecene; ethyl benzene; phenols }\end{array}$ \\
\hline Pungent & Pentan-1-ol; 2-methyl-4-pentenal; buthyl acetate; phenols \\
\hline
\end{tabular}

'apple' were correlated with ethyl acetate, trans-2-pentenal, 1penten-3-ol, pentanol-1-ol, cis-2-penten-1-ol, cis-3-hexen-1-ol, pentan-3-one, trans-2-hexen-1-ol, octan-1-ol, 2-methyl-propan1 -ol and ethanol; the 'taste' notes of 'pungent' and 'astringency' were correlated with ethyl acetate, ethanol, 1-penten-3-ol and pentan-1-ol. Some of these correlations confirm previous results
(Tura et al., 2002): 'lawn' correlated with 1-penten-3-ol, 'banana' with cis-2-penten-1-ol, 'apple' with cis-3-hexen-1-ol and ethanol, and 'butter' with $n$-octane. In this way, many correspondences were found between sensory notes and chemical compounds, most of them in agreement with the literature: Burdock (2002), Kalua et al. (2007), Angerosa et al. (2004) and Morales et al. (2005)

Table 9

Relationships among aromatic chemical compounds and sensory notes with odor and taste thresholds found by several authors (Burdock, 2002; Kalua et al., 2007; Morales et al., 2005)

\begin{tabular}{|c|c|c|}
\hline Chemical compound & Sensory descriptor & Odor/taste thresholds $(\mathrm{mg} / \mathrm{kg})$ \\
\hline \multicolumn{3}{|l|}{ Alcohols } \\
\hline Ethanol & Alcohol, apple, sweet, winey & 30/n.f. \\
\hline 2-Methyl-propan-1-ol & Green & - \\
\hline 3-Methyl-butan-1-ol & Sweet, undesirable, whiskey, woody, yeast & $0.1 /$ n.f. \\
\hline Pentan-1-ol & Balsamic, fruity, pungent, ripe fruit, sticky, strong & $0.47-3 /$ n.f. \\
\hline 1-Penten-3-ol & Butter, fruity, green, hay, lawn, soft green, undesirable, wet earth & $0.4 / 15$ \\
\hline cis-2-Penten-1-ol & Almond, banana, fruity, grass, green & $0.25 / \mathrm{n} . \mathrm{f}$. \\
\hline Hexan-1-ol & Banana, fruity, soft, tomato, undesirable ${ }^{a}$ & $0.4 /$ n.f. \\
\hline trans-2-Hexen-1-ol & Apple, flowers, fruity, grass, green, leaves, sweet, undesirable ${ }^{a}$ & $5-8 / 30$ \\
\hline cis-3-Hexen-1-ol & Apple, banana, fresh, grass, green, leaf & $0.070-1.1-6 / 30$ \\
\hline Octan-1-ol & Green, fusty, musty, sweet, waxy & $0.042-0.480 / 2$ \\
\hline \multicolumn{3}{|l|}{ Aldehydes } \\
\hline 2-Methyl-butanal & Apple, malty, pungent & $0.0052 /$ n.f. \\
\hline 3-Methyl-butanal & Apple, fruity, malty, ripe fruit, sweet & $0.0054 /$ n.f. \\
\hline trans-2-Pentenal & Almond, apple, bitter, fruity, green, ripe fruit, soft fruit & $0.0015-0.3 / 20$ \\
\hline Hexanal & Apple, banana, grass, green, green fruit, sweet & $0.004-0.02-0.08-0.4 /$ n.f. \\
\hline trans-2-Hexenal & Almond, apple, astringent, bitter, fruity, green, lawn, leaf, sweet & $0.030-0.42-1.125 / 10$ \\
\hline \multicolumn{3}{|l|}{ Ketones } \\
\hline Pentan-3-one & Fruity, green, sweet & 70/n.f. \\
\hline 1-Penten-3-one & Bitter, green, mustard, pungent, strawberry, sweet, tomato & $0.001-0.013-0.050 /$ n.f. \\
\hline \multicolumn{3}{|l|}{ Others } \\
\hline Ethyl acetate & Aromatic, bitter, fruity, pleasant, pungent, sticky, sweet, undesirable & $0.005-0.94-5 / 100$ \\
\hline$n$-Octane & Butter, sweet & $0.94 /$ n.f. \\
\hline Acetic acid & Pungent, sour, strong, vinegary & $0.124-0.5-10-60-522 /$ n.f. \\
\hline Phenols & Astringency, bitter, pungent, strong, sweet, walnut husk & 5.5/n.f. \\
\hline
\end{tabular}

n.f., threshold not found.

a An high concentration of chemical compound gives an undesirable sensory note. 
(Tables 8 and 9). Most of the volatile and phenolic compounds showed an average content higher than odor and taste thresholds, explaining the correlations to sensory attributes. Other volatile compounds, correlated to sensory attributes, showed concentrations different from odor and taste thresholds: pentan-3-one had a content lower than odor threshold; trans-2-pentanal, ethyl acetate and octan-1-ol showed a concentration higher than odor, but smaller than taste threshold; pentan-1-ol concentration was both higher and smaller than two odor thresholds found in literature; no thresholds were found for 2-methyl-propan-1-ol odor and/or taste. These volatiles were correlated to the sensory notes even if their concentration was smaller than a given thresholds, this could be due to: a synergic effect with other compounds for that specific sensory note; the odor threshold is possibly more effective than that taste; furthermore, it could be that only in few oils the content was over the thresholds, but the correlation was so strong that was general attributed to all samples.

\section{Conclusions}

The study carried for 4 years on 18 olive local cultivars grown in the same orchards has shown that the aromatic quality of virgin olive oil depends on the cultivar (genetic factor), this has been found particularly for some volatile compounds (ethanol, 2methyl-propan-1-ol, pentan-1-ol, cis-2-penten-1-ol, cis-3-hexen1-ol and octan-1-ol) and sensory attributes ('flowers', 'banana', 'apple', 'walnut', 'hay', 'butter', 'sweet', 'floral' and 'fruity' notes).

The oils from 'Casaliva 1', 'Casaliva 2' and 'Raza' showed a similar aromatic profile, in addition to genetic and chemometric similarity (Bassi et al., 2002). Furthermore, this research has characterized some monovarietal oils from local and minor, underutilized cultivars with a peculiar flavor profile, e.g. 'Casaliva 1', 'Cornarol', 'Grignano', 'Regina' and 'Trepp'. In particular, 'Regina' besides very good oil attributes (Tura et al., 2007), showed some positive horticultural traits (Bassi et al., 2003), although being scarcely cultivated.

Dissecting olive oil quality parameters could be a powerful tool in order to improve our understanding of oil quality, particularly in local and underutilized minor cultivars. They could thus be employed in the new orchards in order to take advantage of their positive horticultural and oil quality traits. This could be particularly crucial for the PDO oils, where the mere geographical origin could not be enough in improving olive oil characterization and consumption, especially if sensorial and/or nutritional attributes are not differentiated within a standard commercial commodity.

\section{Acknowledgements}

The research was funded by the EU Commission, Regulation EC no. 528/99, in co-operation with the Agriculture Department of Lombardy Region, Italy.

The authors wish to thank the olive oil panel tasting groups of Pescara (CRA, Centro Centro di ricerca per l'olivicoltura e l'industria olearia), Savona (O.N.A.O.O.) and Brescia (A.I.P.O.L.).

\section{References}

Alfei, B., 2004. Brescia, two PDO on lakeshores. Olivo Olio 10, 12-14 (in Italian). Angerosa, F., Di giacinto, L., d'Alessandro, N., 1997. Quantitation of some flavo components responsible for the "green" attributes in virgin olive oils. J. High Resolut Chromatogr. 20, 507-510.

Angerosa, F., Servili, M., Selvaggini, R., Taticchi, A., Esposto, S., Montedoro, G.F., 2004 Review. Volatile compounds in virgin olive oil: occurrence and their relationship with quality. J. Chromatogr. A 1054, 17-31.

Baccouri, B., Temime, S.B., Campeol, E., Cioni, P.L, Daoud, D., Zarrouk, M. 2007. Application of solid-phase microextraction to the analysis of volatile compounds in virgin olive oils from five new cultivars. Food Chem. 102, 850-856.
Bassi, D., Failla, O., Pedò, S., Tura, D., Mariani, L., Minelli, R., Geuna, F., Gigliotti, C. 2003. The olive cultivars in Lombardy: agronomic proprieties and oil charac teristics. Quaderni della ricerca no. 25, Regione Lombardia, Italy, http://www.agricoltura regione.lombardia.it/sito/tmpl action asp?Documentold=1292\&SezioneId $=2812000000$ \&action=Documento (in Italian).

Bassi, D., Tura, D., Geuna, F., Failla, O., Pedò, S., 2002. Characterisation of local olive (Olea europea L.) accessions by oil composition, morphological and molecular markers methods. Acta Hortic. 586, 57-60.

Berlioz, B., Cordella, C., Cavalli, J.F., Lizzani-Cuvelier, L., Loiseau, A.M., Fernandez, X., 2006. Comparison of the amounts of volatile compounds in French Protected Designation of Origin virgin olive oils. J. Agric. Food Chem. 54, 10092-10101.

Burdock, G.A., 2002. Fenaroli's Handbook of Flavor Ingredients, 4th ed. CRC Press.

Cavalli, J.F., Fernandez, X., Lizzani-Cuvelier, L., Loiseau, A.M., 2003. Comparison of static headspace, headspace solid phase microextraction, headspace sorptive extraction, and direct thermal desorption techniques on chemical composition of French olive oils. J. Agric. Food Chem. 51, 7709-7716.

Commission Regulation (EC) no. 1989/2003, November 6, 2003. Amending Regulation (EEC) no. 2568/91 on the characteristics of olive oil and olive-pomace oil and on the relevant methods of analysis. Offic. J. Eur. Union L295, 57-77.

Commission Regulation (EC) no. 796/2002, May 6, 2002. Amending Regulation (EEC) no. 2568/91 on the characteristics of olive oil and olive-pomace oil and on the relevant methods of analysis and the additional notes in the Annex to Council Regulation (EEC) no. 2658/87 on the tariff and statistical nomenclature and on the Common Customs Tariff Offic. J. Eur. Union L128, 8-28.

Contini, M., Esti, M., 2006. Effect of the matrix volatile composition in the headspace solid-phase microextraction analysis of extra virgin olive oil. Food Chem. 94 143-150.

Dhifi, W., Angerosa, F., Serraiocco, A., Oumar, I., Hamrouni, I., Marzouk, B., 2005. Virgin olive oil aroma: characterization of some Tunisian cultivars. Food Chem. 93, 697-701.

Galli, C., Visioli, F., 1999. Antioxidant and other activities of phenolics in olives/olive oil, typical components of the mediterranean diet. Lipids 34 (supplement), S23s26.

Garcia-Gonzalez, D.L., Barie, N., Rapp, M., Aparicio, R., 2004. Analysis of virgin olive oil volatiles by a novel electronic nose based on a miniaturized SAW sensor array coupled with SPME enhanced headspace enrichment. J. Agric. Food Chem. 52, 7475-7479.

Haddada, F.M., Manai, H., Daoud, D., Fernandez, X., Lizzani-Cuvelier, L., Zarrouk, M. 2007. Profiles of volatile compounds from some monovarietal Tunisina virgin olive oils. Comparison with French PDO. Food Chem. 103, 467-476.

Harwood, J., Aparicio, R. (Eds.), 2000. Handbook of Olive Oil: Analysis and Properties. Aspen Publishers, Inc., Gaithersburg, Maryland.

Jimenez, A., Aguilera, M.P., Beltran, G., Uceda, M., 2006. Application of solid-phase microextraction to virgin olive oil quality control. J. Chromatogr. A 1121, 140 144.

Kalua, C.M., Allen, M.S., Bedgood Jr., D.R., Bishop, A.G., Prenzler, P.D., Robards, K. 2007. Olive oil volatile compounds, flavour development and quality. A critical review. Food Chem. 100, 273-286.

Kanavouras, A., Kiritsakis, A., Hernandez, R.J., 2005. Comparative study on volatile analysis of extra virgin olive oil by dynamic headspace and solid phase microextraction. Food Chem. 90, 69-79.

Luna, G., Morales, M.T., Aparicio, R., 2006. Characterisation of 39 varietal virgin olive oils by their volatile compositions. Food Chem. 98, 243-252.

Morales, M.T., Luna, G., Aparicio, R., 2005. Comparative study of virgin olive oil sensory defects. Food Chem. 91, 293-301.

Pedò, S., Tura, D., Failla, O., Bassi, D., 2002. Quality aspect of monovarietal olive oils in northern Italy (Garda lake). In: Proceeding of "Convegno Internazionale di olivicoltura" International Conference on Olive Growing. Spoleto (PG), Italy, 22-23 April, (in Italian), pp. 503-508.

Pedò, S., Tura, D., Failla, O., Bassi, D., 2003. Ripeness and quality and composition indices of monovarietal oils. Informatore Agrario 59, 69-73 (in Italian).

Procida, G., Giomo, A., Cichelli, A., Conte, L.S., 2005. Study of volatile compounds of defective virgin olive oils and sensory evaluation: a chemometric approach. J. Sci. Food Agric. 85, 2175-2183.

Tura, D., Failla, O., Bassi, D., Serraiocco, A., 2002. Sensory and chemical analyses of monovarietal olive oils from lake Garda (northern Italy). Acta Hortic. 586, 595598.

Tura, D., Failla, O., Pedò, S., Gigliotti, C., Bassi, D., Serraiocco, A., in press. Effect of seasonal weather variability on olive oil composition in northern Italy. In: Proceedings of the fifth International Symposium on Olive Growing, Turkey, 27 September-2 October 2004, Acta Hortic.

Tura, D., Failla, O., Pedò, S., Gigliotti, C., Serraiocco, A., Bassi, D., 2005. Climate trends and the quality of extra-virgin olive oil. Informatore Agrario 8, 53-56 (in Italian).

Tura, D., Gigliotti, C., Pedò, S., Failla, O., Bassi, D., Serraiocco, A., 2007. Influence of cultivar and site of cultivation on levels of lipophilic and hydrophilic antioxidants in virgin olive oils (Olea europea L.) and correlations with oxidative stability. Sci. Hortic. 112, 108-119.

Tura, D., Prenzler, P.D., Bedgood Jr., D.R., Antolovich, M., Robards, K., 2004. Varietal and processing effects on the volatile profile of Australian olive oils. Food Chem. 84, 341-349.

Vichi, S., Pizzale, L., Conte, L.S., Buxaderas, S., Lopez-Tamames, E., 2003. Solid-phase microextraction in the analysis of virgin olive oil volatile fraction: characterization of virgin olive oils from two distinct geographical areas of northern Italy. J. Agric. Food Chem. 51, 6572-6577. 